\title{
Effects of Estrogens on the Expression of Caveolin-1 in the Urinary Bladders of Female Rats
}

\author{
Sun-Ouck Kim, Seung Hee Song, Seung-Chul Lee ${ }^{1}$, Kyung Aa Cho², Hyung Suck Kim³, In Sang Hwang, Eu Chang Hwang, \\ Kyung Jin Oh, Dongdeuk Kwon
}

Departments of Urology, ${ }^{1}$ Dermatology, ${ }^{2}$ Biochemistry, and ${ }^{3}$ Forensic Medicine, Chonnam National University Medical School, Gwangju, Korea

\begin{abstract}
Purpose: The purposes of this study were to investigate the effect of hormonal alterations on the expression of caveolin-1 in the urinary bladders of ovariectomized rats and to determine the role of caveolin-1 in the overactivity of the detrusor muscle that occurs with hormonal alterations in rats.

Methods: Female Sprague-Dawley rats were divided into three groups: a control group, a group that underwent bilateral ovariectomy (Ovx), and a group that underwent bilateral ovariectomy followed by subcutaneous injections of $17 \beta$-estradiol (Ovx + Est). After 4 weeks, urodynamic studies were done to measure the contraction interval and contraction pressure. The expression and cellular localization of caveolin-1 were determined by Western blot and immunofluorescence in the urinary bladders of rats. Results: On cystometrograms, the contraction interval was significantly shorter in the Ovx group ( $3.0 \pm 0.3$ minute) than in the control group (5.6 \pm 0.5 minute) but was longer in the Ovx + Est group $(9.2 \pm 0.4$ minute $)(\mathrm{P}<0.05)$. Conversely, the average contraction pressure was higher in the Ovx group $(26.4 \pm 0.48 \mathrm{mmHg})$ than in the control group $(21.8 \pm 0.37 \mathrm{mmHg})$ but was lower in the Ovx + Est group $(23.9 \pm 0.76 \mathrm{mmHg})(\mathrm{P}<0.05)$. Caveolin-1 was expressed in the capillaries, arterioles, and venules. Expression of the protein caveolin-1 was significantly lower after ovariectomy and was restored to control levels after treatment with $17 \beta$-estradiol $(\mathrm{P}<0.05)$.

Conclusions: Hormonal alterations cause a significant change in the expression of caveolin-1, which suggests that caveolin-1 might have a functional role in the overactivity of the detrusor muscle related to hormonal alterations in the urinary bladders of rats.
\end{abstract}

Keywords: Caveolin-1; Bladder; Estrogen; Rats

\section{INTRODUCTION}

Traditionally, the urothelium has been considered to be a simple passive barrier between the urinary tract and urine. Recently, however, the role of the urothelium has changed and it is now understood to be a responsive organ capable of sensing a variety of signals from the urinary bladder [1]. Caveolae are small invaginations of the cell membrane that are thought to play an important role in cell surface signaling and intracellular lipid transport [2]. Since its discovery a decade age, caveolin-1 has been shown to play a role in the regulation of the signal transduction pathway in various endothelial cells and epithelial cells
[3]. Caveolins are major constituents of caveolae [4]. Of the three isoforms of caveolin (caveolin-1, -2, and -3), caveolin-1 has been characterized extensively and shown to interact through its scaffold domain with caveolae-associated signal proteins. The functional roles of caveolin-1 are beginning to emerge with the study of caveolin-1 knockout mice. Mice lacking caveolin-1 also lack caveolae in the bladder and exhibit several urological disorders, including decreased detrusor contractility on stimulation with carbachol $[5,6]$.

Previous experiments have focused on the expression of caveolin in the detrusor muscle of the urinary bladder. No studies to date, however, have investigated the combined changes in
Corresponding author: Sun-Ouck Kim

Department of Urology, Chonnam National University Hospital, Chonnam National University Medical School, 8 Hak 1-dong, Dong-gu, Gwangju 501-757, Korea

Tel: +82-62-220-6705 / Fax: +82-62-227-1643 / E-mail: seinsena@hanmail.net Submitted: February 28, 2011 / Accepted after revision: March 20, 2011
This is an Open Access article distributed under the terms of the Creative Commons Attribution Non-Commercial License (http://creativecommons.org/licenses/by-nc/3.0/) which permits unrestricted non-commercial use, distribution, and reproduction in any medium, provided the original work is properly cited. 
expression of caveolin-1 in the urinary bladder or the functional activity of these proteins in response to hormonal alterations. The purpose of the present study, therefore, was to investigate the effect of estrogen on the expression of caveolin-1 in the urinary bladders of rats, with a focus on suburothelial microvascular expressing caveolin-1.

\section{MATERIALS AND METHODS}

\section{Experimental Model}

Female Sprague-Dawley rats (12 weeks old, 230-240 g, $\mathrm{n}=90$ ) were divided into three groups: a control group $(n=30)$, a group that underwent bilateral ovariectomy $(\mathrm{Ovx}, \mathrm{n}=30)$, and a group that underwent bilateral ovariectomy plus subcutaneous injection with 17 $\beta$-estradiol (Ovx+Est, $\mathrm{n}=30$; Sigma-Aldrich Co., St. Louis, MO, USA). The control group underwent a sham operation. The Ovx group underwent a bilateral ovariectomy and was treated with an oil vehicle. The Ovx + Est group underwent a bilateral ovariectomy, which was followed by daily treatment with subcutaneous injections of estradiol (50 mg/kg/day) for 7 days after ovariectomy. All experimental animals were fed a standard diet up until the day before the experiment. Four weeks after ovariectomy and 3 weeks after hormonal replacement, animals with an estrous cycle confirmed via a vaginal smear were premedicated with xylazine $(2.2 \mathrm{mg} / \mathrm{kg}$ intramuscularly) and anesthetized with a zolazepam/tiletamine cocktail ( $4.4 \mathrm{mg} / \mathrm{kg}$ intramuscularly). The study was approved by the Ethics Committee of the Chonnam National University Medical School.

\section{Cystometrogram}

Four weeks after the operation, rats ( $\mathrm{n}=10$ in each group) were anesthetized with a subcutaneous injection of $1.2 \mathrm{~g} / \mathrm{kg}$ urethane. A suprapubic midline incision was performed to expose the bladder, a transvesical catheter with a fire-flared tip (polyethylene catheter-50) was inserted into the dome of the bladder and secured with a ligature, and the abdomen was closed. The catheter was connected to a pressure transducer and syringe pump via a 3-way stopcock to record intravesical pressure and to infuse saline into the bladder. After the bladder was emptied, cystometry was performed with saline infused at a rate of $0.04 \mathrm{~mL} /$ min. The contraction pressure and contraction interval were recorded.

\section{Western Blot}

All minced tissues were homogenized in ice-cold isolation solu- tion with a Tissumizer homogenizer (Teledyne Tekmar, Mason, $\mathrm{OH}, \mathrm{USA}$ ). Tissues were homogenized with five bursts of five strokes of a micro-sawtooth generator. Tissue homogenates $(\mathrm{n}=$ 10 in each group, $50 \mu \mathrm{g}$ of protein) were separated by $12 \%$ sodium dodecyl sulfate-polyacrylamide gel electrophoresis and transferred to polyvinylidene fluoride membranes (Amersham Pharmacia Biotech, Buckinghamshire, UK). The blots were then washed with tris-buffered saline Tween-20 (10 mM Tris-HCl, pH 7.6; $150 \mathrm{mM} \mathrm{NaCl}$; and $0.05 \%$ Tween-20). The membrane was blocked with 5\% skim milk for 1 hour and incubated with the appropriate primary antibody. Monoclonal mouse antibodies for caveolin-1 (1:2,000; Chemicon, Ramona, CA, USA) and a polyclonal rabbit antibody against glyceraldehyde 3-phosphate dehydrogenase (GAPDH) $(1: 4,000$; Cell Signaling Technology Inc., Danvers, MA, USA) were used. The membrane was then washed, and caveolin-1 and GAPDH were detected with goat anti-mouse-IgG and goat anti-rabbit-IgG conjugated to horseradish peroxidase, respectively. The antibodies were incubated at $4^{\circ} \mathrm{C}$. The bands were visualized by enhanced chemiluminescence (Amersham Pharmacia Biotech). GAPDH was used as an internal control. Densitometry analysis was performed with a Studio Star Scanner with the use of National Institutes of Health image V1-57 software.

\section{Immunofluorescence Staining}

The tissue sections ( $\mathrm{n}=10$ in each group, 10 sections in each tissue) were rinsed in phosphate-buffered saline (PBS) and then treated with normal chicken serum for 30 minutes to block nonspecific binding. After being washed in PBS, the sections were incubated with antibodies for caveolin-1 (Chemicon) in PBS for 12 to 14 hours at $4^{\circ} \mathrm{C}$. Immunoreactivity for caveolin-1 was detected with the use of Alexa Fluor 488 chicken anti-mouse IgG (H+L; Invitrogen, Carlsbad, CA, USA). Tissues were mounted with the use of mounting solution containing 4'-6-diamidino-2-phenylindole. For a negative control, tissues were prepared in a similar manner, except that caveolin-1 was omitted from the incubation solution. Tissues were examined with an LSM 510 confocal microscope (Carl Zeiss, Seoul, Korea) with an excitation wavelength appropriate for Alexa Fluor $(405 \mathrm{~nm})$ and Alexa Fluor (488 nm). Final images were constructed with the use of LSM Image Examiner software.

\section{Statistical Analysis}

The results are expressed as means \pm standard deviations; however, the data for the cystometric parameters are expressed as 
means \pm standard errors of the mean. Analysis of variance was used to test the null hypothesis that there would be no differences in the mean expression levels between the three groups. Differences were considered significant at $\mathrm{P}<0.05$.

\section{RESULTS}

All of the animals survived for 4 weeks after surgery. Body weight was significantly higher in the Ovx group $(366 \pm 9.7 \mathrm{~g})$ than in the control group $(263 \pm 12.1 \mathrm{~g})$. Treatment of ovariectomized animals with $17 \beta$-estradiol reduced the body weight to the control level $(264 \pm 11.3)(\mathrm{P}<0.05)$. No significant differences in bladder weight were observed between the groups.

\section{Effect of Estrogen on the Cystometric Parameters}

On cystometrograms performed 4 weeks after the operation, the contraction interval was significantly lower in the Ovx group (3.0 \pm 0.3 minute) than in the control group ( $5.6 \pm 0.5$ minute) but was higher after estrogen treatment $(9.2 \pm 0.4$ minute $)(\mathrm{P}<$ $0.05)$. Conversely, the average contraction pressure was higher in the Ovx group $(26.4 \pm 0.48 \mathrm{mmHg})$ than in the control group $(21.8 \pm 0.37 \mathrm{mmHg})$ but was lower after estrogen treatment (23.9 $\pm 0.76 \mathrm{mmHg})(\mathrm{P}<0.05)$ (Fig. 1).

\section{Effect of Estrogen on the Expression of Caveolin -1}

The expression of caveolin-1 was easily detected and was ex- pressed in capillaries, venules, and arterioles in the subepithelial layer (Fig. 2). Immunofluorescence showed that, in terms of the cellular patterns of labeling, the expression of caveolin-1 in the control group was similar to that in the Ovx and Ovx + Est groups (Fig. 2). Western blot analysis showed 22-kDa bands corresponding to caveolin-1 protein (Fig. 3). Caveolin-1 protein was recognized in all groups. Caveolin-1 protein expression decreased significantly after ovariectomy. However, the expression of this protein was restored to the control level after treatment with $17 \beta$-estradiol $(\mathrm{P}<0.05 ;$ Fig. 3$)$.

\section{DISCUSSION}

This study showed an altered expression of caveolin-1 in the bladder after ovariectomy and estrogen replacement in the urinary bladders of female rats. Four weeks after surgery, cystometric results showed that the voiding interval was shorter and the voiding pressure was higher in the bladders of ovariectomized rats than in the controls and returned to the normal controls value after the rats were treated with estrogen. The immunohistochemical study showed that the localization of caveolin-1 in the urinary bladders was detected in the capillaries, venules, and arterioles of the subepithelial layers. These results provide evidence that estrogen deprivation induces bladder instability, and caveolin-1 may play a role in the bladder dysfunction induced by hormonal changes in females. To our knowl-
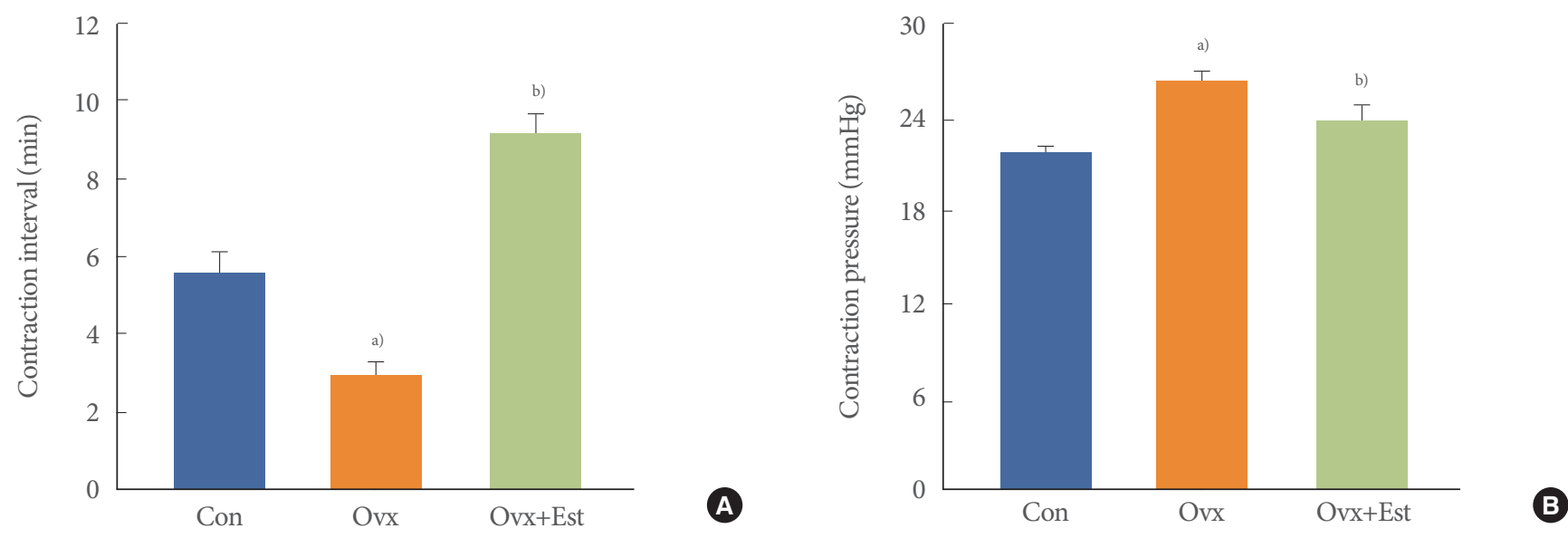

Fig. 1. Representative urodynamic profiles of three groups of rats: a control group (Con), a group that underwent bilateral ovariectomy (Ovx), and a group that underwent bilateral ovariectomy followed by subcutaneous injections of $17 \beta$-estradiol (Ovx + Est). (A) The contraction interval in the Ovx group was significantly shorter than that in the control group $\left({ }^{(a)} \mathrm{P}<0.05\right)$ and was restored to the control value after estrogen treatment $\left.{ }^{(b)} \mathrm{P}<0.05\right)$. (B) Note the increased peak pressures with each voiding contraction in the Ovx group and recovery to the control value in the Ovx + Est group. The lower panels denote the means \pm standard deviations of 10 experiments for each condition determined by cystometrogram. ${ }^{\text {a) }} \mathrm{P}<0.05$ vs. Con. ${ }^{\text {b) }} \mathrm{P}<0.05$ vs. Ovx. 


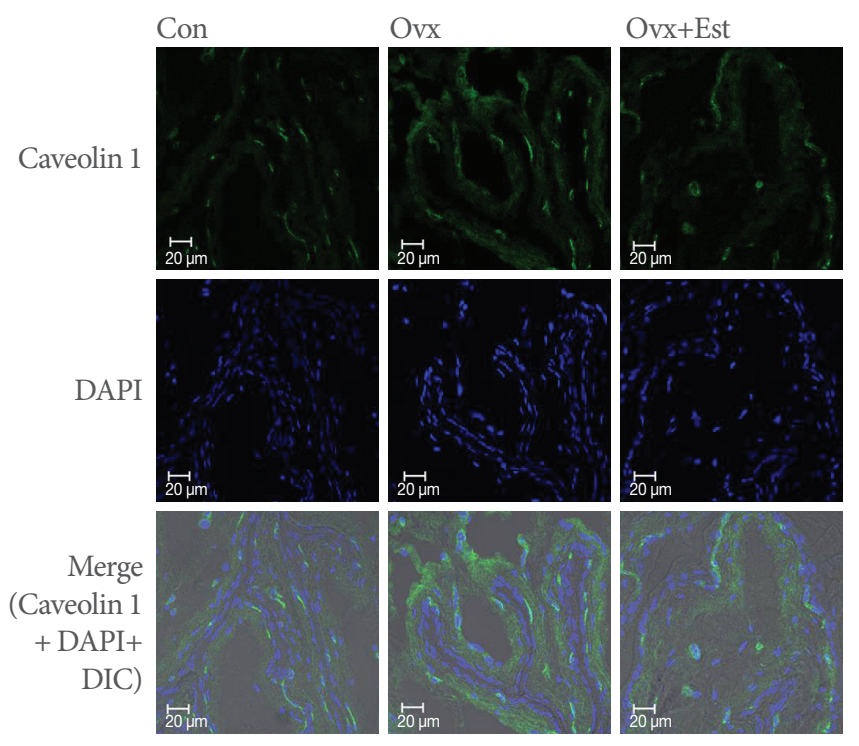

Fig. 2. Immunofluorescence labeling for caveolin-1 in the urinary bladders of three groups of rats: a control (Con) group, a group that underwent bilateral ovariectomy (Ovx), and a group that underwent bilateral ovariectomy followed by subcutaneous injections of $17 \beta$-estradiol (Ovx +Est). Caveolin-1 expression (green) was noted throughout the capillaries, arterioles, and venules. Immunofluorescence showed that, in terms of the cellular patterns of labeling, the expression of caveolin-1 in the Con group was similar to that in the Ovx and Ovx + Est groups. The horizontal scale bar at the bottom left of each panel indicates the magnification power. DAPI, 4,6- diamidino-2-phenylindole; DIC, differential interference contrast.

edge, this is the first study to show the possible occurrence of signaling in the suburothelial microvasculature, via caveolin-1, in an ovariectomized rat model in which bladder dysfunction was induced.

Urinary tracts are sensitive to female sex steroids. Many studies have shown that ovariectomy and estrogen administration induce pronounced alterations in lower urinary tract function [7]. Ovariectomy results in atrophy of the bladder mucosa, decreased smooth muscle mass of the detrusor, decreased bladder compliance, and decreased detrusor contractility [8].

Traditionally, the urothelium has been considered to be a simple passive barrier between the urinary tract and urine. Recently, however, the role of the urothelium has changed and it is now understood to be a responsive organ capable of sensing a variety of signals from the urinary bladder [1].

Evidence suggests that the urothelium can mediate water and solute transport under certain conditions in some animals $[9,10]$. Many studies have shown that urinary bladder epithelium con-
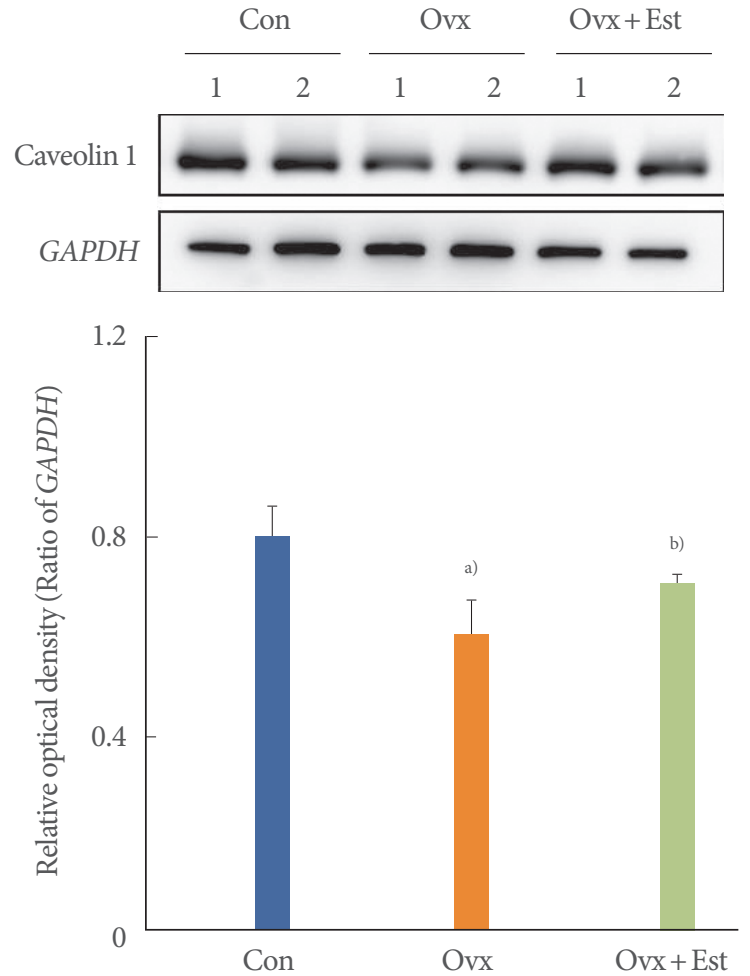

Fig. 3. Immunoblotting for caveolin-1 in urinary bladder tissue from three groups of rats: a control (Con) group, a group that underwent bilateral ovariectomy (Ovx), and a group that underwent bilateral ovariectomy followed by subcutaneous injections of $17 \beta$-estradiol (Ovx +Est). The anti-caveolin- 1 antibodies recognize the $22-\mathrm{kDa}$ bands. The anti-glyceraldehyde 3phosphate dehydrogenase (GAPDH) antibody recognizes the $42-\mathrm{kDa}$ band. The expression of caveolin-1 protein was significantly lower in the Ovx group. However, this expression was restored to the control level after treatment with $17 \beta$-estradiol in the Ovx +Est group. The lower panels denote the means \pm standard deviations of the 10 experiments for each condition, as determined by densitometry relative to $G A P D H .{ }^{\text {a) }} \mathrm{P}<0.05$ vs. Con. ${ }^{\text {b) }} \mathrm{P}<0.05$ vs. Ovx.

tains a sodium transporter [11], which has been described as an epithelial sodium channel (ENaC) [12]. ENaC has been known to be responsible for salt and fluid transport across the epithelia of many tissues. Araki et al. [13] investigated the role of ENaC in bladder dysfunction in male patients with clinically detected bladder outlet obstruction. They reported that the expression of $\mathrm{ENaC}$ was significantly greater in the bladders of these patients than in the bladders of a control group and that $\mathrm{ENaC}$ expression correlated with the patients' storage symptom scores.

Caveolae were discovered more than 50 years ago and were described as 50-100-nm subcellular structures occurring in cap- 
illary endothelial cell [14]. Caveolae are plasma membrane invaginations that play a role in signaling [15]. Several important cellular processes-such as regulation of lipid transport, cholesterol homeostasis, endocytosis, and transcytosis in endothelial and epithelial cells-have been attributed to these microdomains [15]. Caveolin is essential for the formation of caveolae. The caveolin protein family consists of three isoforms (caveolin-1, caveolin-2, and caveolin-3), each of which has a specific expression pattern in particular cell types. Endothelial cells and fibroblasts express high levels of caveolin-1 and caveolin-2, whereas striated and cardiac muscles express mostly cavelin-3 [16,17]. Mice lacking caveolin-1 also lack caveolae in the bladder and exhibit several urologic disorders, including decreased detrusor contractility on stimulation with carbachol $[5,6]$ which is somewhat contrary result in terms of bladder contractility related with caveolin compared to our study result. Genetic ablation of caveolae was found to be associated with a $70 \%$ decrease in acetylcholine release from bladder nerve terminals [5]. It has been reported that aging and pregnancy are associated with a reduction in caveolae in the urinary bladder [18,19]. Furthermore, a few caveolae can be detected in hypertrophied bladder smooth muscle induced by bladder outlet obstruction, which highlights the dynamic nature of caveolae in the urinary bladder. However, the functional significance of these structures in relation to the physiology of the micturition response and voiding function had not been fully understood until now. More recently, Cristofaro et al. [20] reported that caveolin has a central role in the regulation of G-protein-coupled signaling pathways in smooth muscle contraction of the bladder. In a recent study of the influence of hormones on the expression of caveolin-1 in the urinary bladders of rats, Zhu et al. [21] reported that estrogen replacement increased the amount of caveolae and caveolin-1 protein in the urinary bladder.

However, no studies have yet investigated the expression of caveolin-1 in the suburothelial microvascular endothelial cells of the urinary bladder or the changes in the functional activity of these proteins in response to changes in sex hormones. In the present study, the expression of caveolin-1 in the urinary bladder was significantly affected by ovariectomy and estrogen replacement and was associated with the improvement and recovery of cystometric parameters. These findings suggest that caveolin-1 is influenced by the bladder dysfunction induced by hormonal alterations. One possible reason for this influence on caveolin-1 expression is the location of caveolin-1, i.e., the microvasculature is highly dependent on hormonal alterations. A decreased blood supply in the urinary bladder has been reported after estrogen deprivation. Our results suggest that ovariectomy may lead to a significant down-regulation of caveolin-1 expression in the urinary bladder of rats, which is presumptive evidence that caveolin-1 is involved in the lower urinary tract symptoms induced by hormonal alterations, probably via modifications in specific cellular transmission and signaling pathways. A limitation of our study was that the precise functional activity of caveolin-1 was not fully elucidated. However, we did show a change in caveolin- 1 expression in the urinary bladders of ovariectomized rats and the possible role of caveolin- 1 in the overactivity of the detrusor muscle related to hormonal alterations in the urinary bladders of rats. Additional studies are needed to investigate the expression and localization of all members of the caveolin family in the urinary bladder and their related functional role in the underlying mechanisms of bladder pathophysiology and dysfunction.

In conclusion, this study showed that detrusor overactivity induced by estrogen deprivation causes a significant decrease in the expression of caveolin-1 in the urinary bladders of female rats. This finding suggests that the caveoln-1 isoform might change the signal activity in the bladder and might have a functional role in the detrusor overactivity that occurs in association with hormonal alterations.

\section{CONFLICT OF INTEREST}

No potential conflict of interest relevant to this article was reported.

\section{ACKNOWLEDGEMENTS}

This research was supported by the Basic Science Research Program through the National Research Foundation of Korea (NRF) funded by the Ministry of Education, Science and Technology (2009-0069443, 2010-1372, 2010-2296), and by grant CRI1107922, Chonnam National University Hospital Research Institute of Clinical Medicine.

\section{REFERENCES}

1. de Groat WC. The urothelium in overactive bladder: passive bystander or active participant? Urology 2004;64(6 Suppl 1):7-11.

2. Smart EJ, Ying Y, Donzell WC, Anderson RG. A role for caveolin in transport of cholesterol from endoplasmic reticulum to plasma 
membrane. J Biol Chem 1996;271:29427-35.

3. Razani B, Lisanti MP. Caveolin-deficient mice: insights into caveolar function human disease. J Clin Invest 2001;108:1553-61.

4. Souto RP, Vallega G, Wharton J, Vinten J, Tranum-Jensen J, Pilch PF. Immunopurification and characterization of rat adipocyte caveolae suggest their dissociation from insulin signaling. J Biol Chem 2003;278:18321-9.

5. Lai HH, Boone TB, Yang G, Smith CP, Kiss S, Thompson TC, et al. Loss of caveolin-1 expression is associated with disruption of muscarinic cholinergic activities in the urinary bladder. Neurochem Int 2004;45:1185-93.

6. Lai HH, Boone TB, Thompson TC, Smith CP, Somogyi GT. Using caveolin-1 knockout mouse to study impaired detrusor contractility and disrupted muscarinic activity in the aging bladder. Urology 2007;69:407-11.

7. Aikawa K, Sugino T, Matsumoto S, Chichester P, Whitbeck C, Levin $\mathrm{RM}$. The effect of ovariectomy and estradiol on rabbit bladder smooth muscle contraction and morphology. J Urol 2003;170(2 Pt 1): 634-7.

8. Lin AD, Levin R, Kogan B, Whitbeck C, Chichester P, Sokol R, et al. Estrogen induced functional hypertrophy and increased force generation of the female rabbit bladder. Neurourol Urodyn 2006; 25:473-9.

9. Negrete HO, Lavelle JP, Berg J, Lewis SA, Zeidel ML. Permeability properties of the intact mammalian bladder epithelium. Am J Physiol 1996;271(4 Pt 2):F886-94.

10. Cross WR, Eardley I, Leese HJ, Southgate J. A biomimetic tissue from cultured normal human urothelial cells: analysis of physiological function. Am J Physiol Renal Physiol 2005;289:F459-68.

11. Eaton DC. Intracellular sodium ion activity and sodium transport in rabbit urinary bladder. J Physiol 1981;316:527-44.
12. Smith PR, Mackler SA, Weiser PC, Brooker DR, Ahn YJ, Harte BJ, et al. Expression and localization of epithelial sodium channel in mammalian urinary bladder. Am J Physiol 1998;274(1 Pt 2):F91-6.

13. Araki I, Du S, Kamiyama M, Mikami Y, Matsushita K, Komuro M, et al. Overexpression of epithelial sodium channels in epithelium of human urinary bladder with outlet obstruction. Urology 2004; 64:1255-60.

14. Palade GE. An electron microscope study of the mitochondrial structure. J Histochem Cytochem 1953;1:188-211.

15. Cohen AW, Hnasko R, Schubert W, Lisanti MP. Role of caveolae and caveolins in health and disease. Physiol Rev 2004;84:1341-79.

16. Razani B, Woodman SE, Lisanti MP. Caveolae: from cell biology to animal physiology. Pharmacol Rev 2002;54:431-67.

17. Song KS, Scherer PE, Tang Z, Okamoto T, Li S, Chafel M, et al. Expression of caveolin-3 in skeletal, cardiac, and smooth muscle cells. Caveolin- 3 is a component of the sarcolemma and co-fractionates with dystrophin and dystrophin-associated glycoproteins. J Biol Chem 1996;271:15160-5.

18. Elbadawi A, Yalla SV, Resnick NM. Structural basis of geriatric voiding dysfunction. II. Aging detrusor: normal versus impaired contractility. J Urol 1993;150(5 Pt 2):1657-67.

19. Bakircioglu ME, Sievert KD, Lau A, Lin CS, Lue TF. The effect of pregnancy and delivery on the function and ultrastructure of the rat bladder and urethra. BJU Int 2000;85:350-61.

20. Cristofaro V, Peters CA, Yalla SV, Sullivan MP. Smooth muscle caveolae differentially regulate specific agonist induced bladder contractions. Neurourol Urodyn 2007;26:71-80.

21. Zhu Q, Resnick NM, Elbadawi A, Kuchel GA. Estrogen and postnatal maturation increase caveolar number and caveolin-1 protein in bladder smooth muscle cells. J Urol 2004;171:467-71. 